\title{
SUL MOVIMENTO INIZIALE DELLE ONDE SISMICHE REGISTRATE $\Lambda$ ROMA DURANTE IL PERIODO 1938-1943
}

\author{
D. Di Filippo - L. Mancelit
}

E lien nola l'importanza che ha, nello studio di un terremoto, la conoseenza del senso dello spostamento del suolo, in corrispondenza delle onde $P$.

Lal direzione del moto, nelle varie stazioni che circondano l'epicentro, da prezione indicazioni sulla natura fisica della callsa della scossa: conoscendo la distribuzione delle compressioni e delle dilalazioni nei vari azimut e a varie distamze, i puio precisare se il terremoto i satto originato da una frattura, da uno sprofondamento (1) da un! sollevamento.

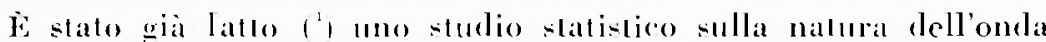
iniziale dei terremoli rewi-laali alla tazione di lace. Tale sludio, comprensivo di un periodo di 14 anni (1910-1914) i stato proseguito

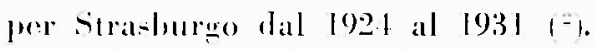

Nel presente lavoro intendiamo procedere in modo analogo per quel che riguarda la stazione simmica di Romal.

Gli strumenti di cui dispone quesa stazione, come is stato altroie descritto (1) sono:

simografi a registrazione oftical componenti N.S, E-W. rerlicale Wilip (a corlo prriodo): componenti N-S, E-W Galitzin a lunga periodol;

simograti a regi-trazione meceanical, tipo Wiecherl: $1000 \mathrm{~kg}$ orizzontale. $1300 \mathrm{~kg}$ verticale, $200 \mathrm{~kg}$ orizzontale, $80 \mathrm{~kg}$ verlicale.

La natura dellonda iniziale cosi conne arriva a Romil, è dunque individuabile senza posibilita di errori.

Per questo primo studio statistleo di simo valsi delle registrazioni relative al quincquennio 1938-43.

Un wran numero di terremoti sono stati onessi perehé, a causa delle difficolta d'ogni sorta renulesi a creare nel periodo bellico, non is stoto posibile individuame gli epicentri. Tutlavia gli elementi che al,biamo a di.posizione sono sufficienti per dare unidea generale della distriluzione delle compresioni e delle dilalazioni. 
La labella n. I si riferisec ai terremoti arvenuti nell'Asia Mi. nore - Mare Eqeo. Qui, come si vede, compressioni c dilatazioni sono mescolate senza aleun carattere di prevalenza. La maggior parte dei teremoli is a pofondià normale: solo quattro su 19 hanno ma profonclita che si agyira sui $100 \mathrm{~km}$.

Un gruppo a parte formano i terremoti della Romania: lre soli Crilevantii, tutti nell'anno 1940, a breve intervallo di lempo l'uno dall'alıo. Il medesimo epicentro, quasi la stessa profondita, la stessa natura dell'omba iniziale, fanno pensare ad 101 unico processo che si s croluto in un tempo abhastanza hreve: cirea 20 giomi.

La labella n, 2 riporta tulli i terremoti, con epicentro indiviluato, arrenuti nell'Asia, per una fascia compresa laa i due meridiani $45^{\circ} \mathrm{E}-130^{\circ} \mathrm{F}$ e i due paralleli $10^{\circ} \mathrm{N}-60^{\circ} \mathrm{N}$. Come si rede, ivi prevalgono netlamente le compressioni: su 13 terremoti di sono solamente tre dilatazioni, due delle quali in corrispondenza di profondita superiori alla normale.

Fel lavoro citato, si era pure trovato che tutti i terremoti provenienti dall'Asia davano al Uecle delle compressioni. Dato che sia Roma che Lecle si trovano ad Orest della zona in esame, si polvelyhe forse derlure che in questi ultimi anni i processi di generazione lei terremoti nell'Asia continentale, sono rimasti immulati.

Ancora come compressioni arrivano a Roma i terremoti provenienti dalla zona delle Filippine, dell’isola di Formosa e delle Marianne, fverli tabella 3 s. Unica eccexione nu teremoto profondisismo $(h=700 \mathrm{~km})$ che presenta invece una dilatazione.

Dal Giappone tabella n. L, zona atlivisima dal punto di visla sismico, arrinano quasi tutte compressioni. Rarissimi wli esempi di dilatazione nei terremoti di profonditi normale folo due stl 20): quasi tulte dilatazioni insece per i terremoti profondi, $i$ quali inoltre risultano distribuiti prevalentemente lungro la costa asiatical.

Anche nella zona delle isole Aleutine prevalgono le compressioni per i terremoti normali, e le dialazioni per quelli profondi (v. Labella n. 5). Seguento ancora l'areo che raceliude loceano Pacifico, e costegriandolo in scuso antiorario, si scende al wruppo delle lsole (Solomone, Nuove Ebridi, Figii, Tonga, Samoa) che si slaccano completamente dal bloceo continentale: ivi compressioni e dilalazioni si distribuiscono in eguale nisura. anche per i terremoti pro. fondi che qui abbondano più che altrove (tabella n. 6).

Nawgiore regolarita si riseontra invece nei terremoti provenien- 
li dall'America del Nord (v. tabella n. 7 per i quali l'impulso iniziale i costantemente una dilatazione, eio che concorda con i rilievi lalli ad licele e Strashurgo.

Questi terremoti, di profondita normale o quasi, risultano tutti distribuiti sulla costa ovest. I terremoti provenienti dall'America Centrale e dal Messico arrirano ad Uecle rome rompressioni; invece per Roma, dall'America Centale, ivi comprese le Anlille, le dilalazioni si mescolano alle compressioni nel rapporto di 1 a 3 : clifatti su 18 teremoti 6 sono dilatazioni (v. tabella n. 8 .

Anche per l'America del Surl le dilatazioni si mantengono all'incirca nello stesso rapporto rispetlo alle compressioni 17 su J9) (r. tahella n. 9): solo rhe qui il numero dei terremoti profondi supera quello dei terremoti normali, e cinque, delle sette diatarioni, compaiono trat essi.

Pochisimi i terrenoli con epicentro in Africas: solo quattro di cui due compreswioni e due dilatazioni (v. tabella n. 10): le due compressioni provengono pero da un medesimo epicentro. Lal scarsitat dei teremoti africani, lutli superficiali, stareble a confortare l’idea dell'Africa rome un bloceo stabile dal quale, secomblo alcune teorie. si sarebbero distacali wli altri continenti.

Una uniformita piutosto eccezionale presenta la zona dell'oceano dilantico d'onde grli impulsi iniziali pervengono a Roma come compressioni: isolo una deloole dilatazione interompe la regrolarital: prli cpicentri sono distributiti quasi tutti sulla cresta mediana Ailantica e sono prevalentemente superficiali: solo 3 . su 12, hanno una profondita variabile la 100 a $250 \mathrm{~km}$.

Dai pochi terremoti provenienti dalleceano Indiano (v. labella 11. I2/ non si puo caratterizare la prevalenza del moto iniziale: in questa zona. dei selte terremoti in questione. uno solo a molto profondo: quello del 2 ! novembe 1939 . e questo presenta una compresione.

Zona pochisimo attiva i l'oceano Antartico (r. tabella n. 13 . fimico terremoto che in einque anni i stato registrato dalla stazione di Roma, si presenta ivi con mal dilatazione.

La rarta di pag. 592 mostra um planisfero a proiezione Mercatore su rui sono traceiate line equidistanti ed isoazimutali. con centro Roma: su esso sono riportati gli epientri di tutti i terremoti elencati nelle precerlenti tabelle con un simbolismo che permette di individuare le comprescioni. Ie dilatazioni o i terremoti profondi. L'esame 


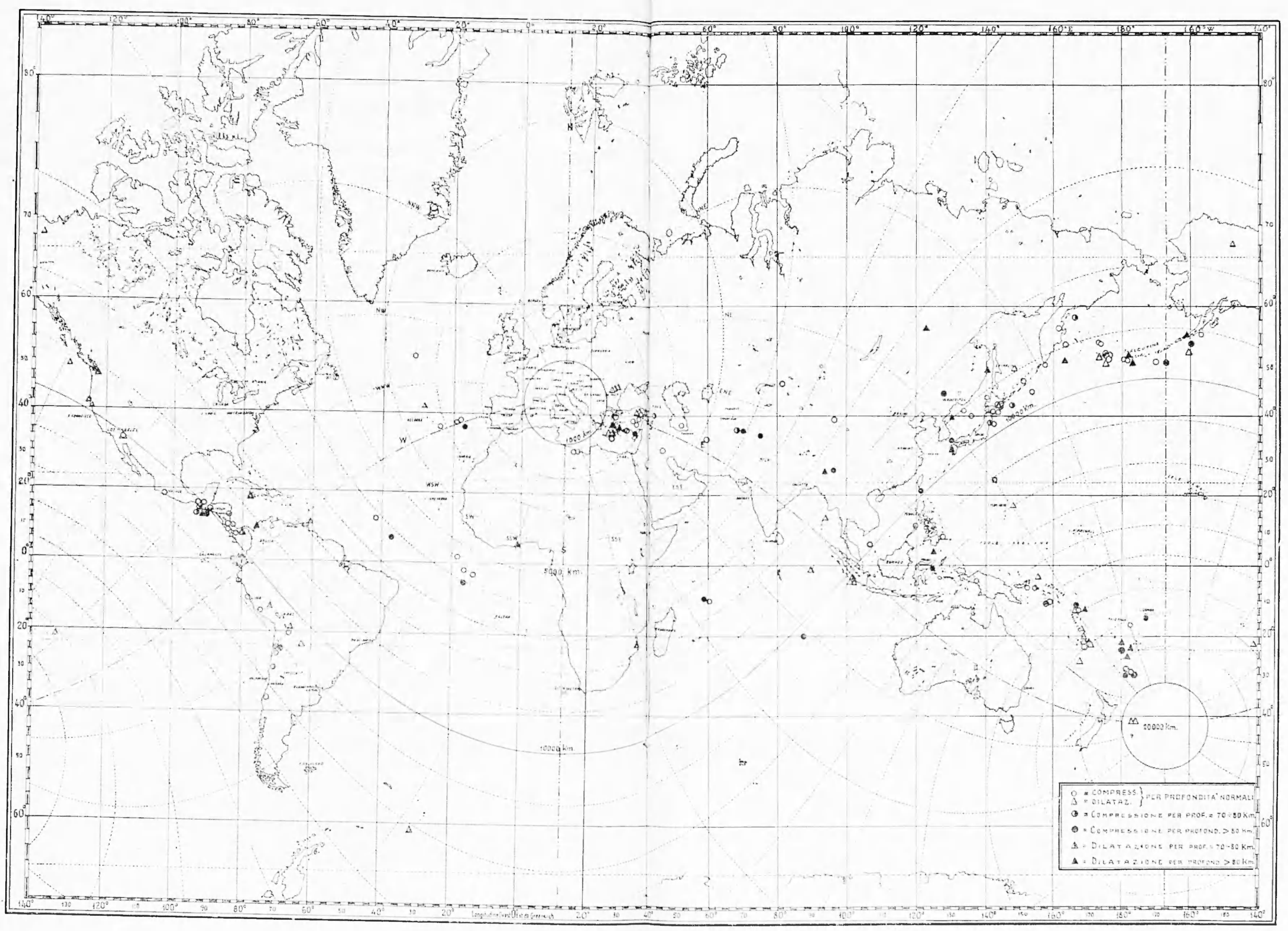


di questa carta permetle di riassmere la siluazione sismiea controllata da Roma, nel modo seguente:

per quanto riguarda il hacino del Pacifico, sembra che si possa asserire che nell'arco occidentale (Filippine, Giappone, Alentine) predominano le compressioni: negli arehi sud-oceidentale, sud, sudorientale igruppo delle Isole, coste dell'America del Sul, Ameriea Contrale, si altemano indifferentemente le compressioni con le dilataxioni, e queste ullime poi predominano nellamente lungro le coste dell'America del Norel.

Lumgro la fascia circumpacifica, zona di grande sismicila, ablsondano i terremoti profondi e profondissimi questo fato, unito alla presenza di molti vuleani, ha dato la possibilita a Tumer, insieme con altre considerazioni, di enunciare la sua fantasiosa ipotesi sulla cicatrice della Luna, che si sarehbe staceala dalla Terra in corrispondenza del Pacifico.

Rari sono i terremoti con epicentro interno ai continenti: -i achlensano prevalentemente sulle coste e sulle isole: unica recezione i terrenoti asiatici che sono invece lutti continentali e si presentano onogeneamente come compressioni fecelto quelli dell'Asia Mlinore)

Pochi pure i terremoti con epicentro in pieno Oceano, lontano dalle coste, se si eccettuano quelli dell'Oceano Alantico, distribuiti peró prevalentemente, come s’e dello, lungo la dorsale mediana.

Dalle lerre comprese nell'angolo X-W, N-E rispetlo a Roma, non pervengono terremoti: cio non siznifica che lale zona sia del lutlo asismiea, perelté slazioni piò a Nord di Roma altestano ma qual

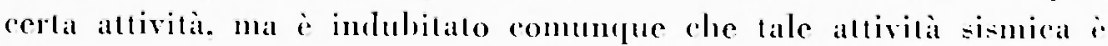
di gran lunga inferiore a cquella di ogni alıra zona.

\section{India, Albania, Grecia o Jugoslatia.}

Una tratlazione a parte va fatla per l'latia e i paesi immediatamente vicini. La fig̣. I mostra la distribuzione degrli epiecntri, per della zona, fno a $1000 \mathrm{~km}$ da Roma. Numerosissimi sono ivi i leree moti, specialnente in Italia: pero essi son lutti, o quasi lutti, superficiali. e poiché nel presente lasoro ci siamo limitati a considerare solamente i terremoti registrati a Roma, i ovvio che la sismicita della zona non $\dot{e}$ in realta quella che appare dalla figura, ma molto piu intensa, come si potreble documentare con le registrazioni che $-i$ otlengono nelle varie stazioni della rele sismica ilaliana.

Dalla tabella n. $1+$ si vede come, sll 35 terremoti con epicentros 
in Italia, 22 siano compressioni e gli altri 13 dilatazioni: se poi si osserva che la maggior parte delle dilatazioni sono di terremoti relalivi alla stessa zona e avvenuti nello stesso torno di tempo (1m1. 27-28: 39.-1(1-41-42-43-44), si rele come le compressioni prevalgano nettamenle.

Per i 9 terremoti fuori d'Italia prevalugono invece le dilatazioni: e queste si trovano tutle ad est di Roma (Grecia, Albania, Jugosla-

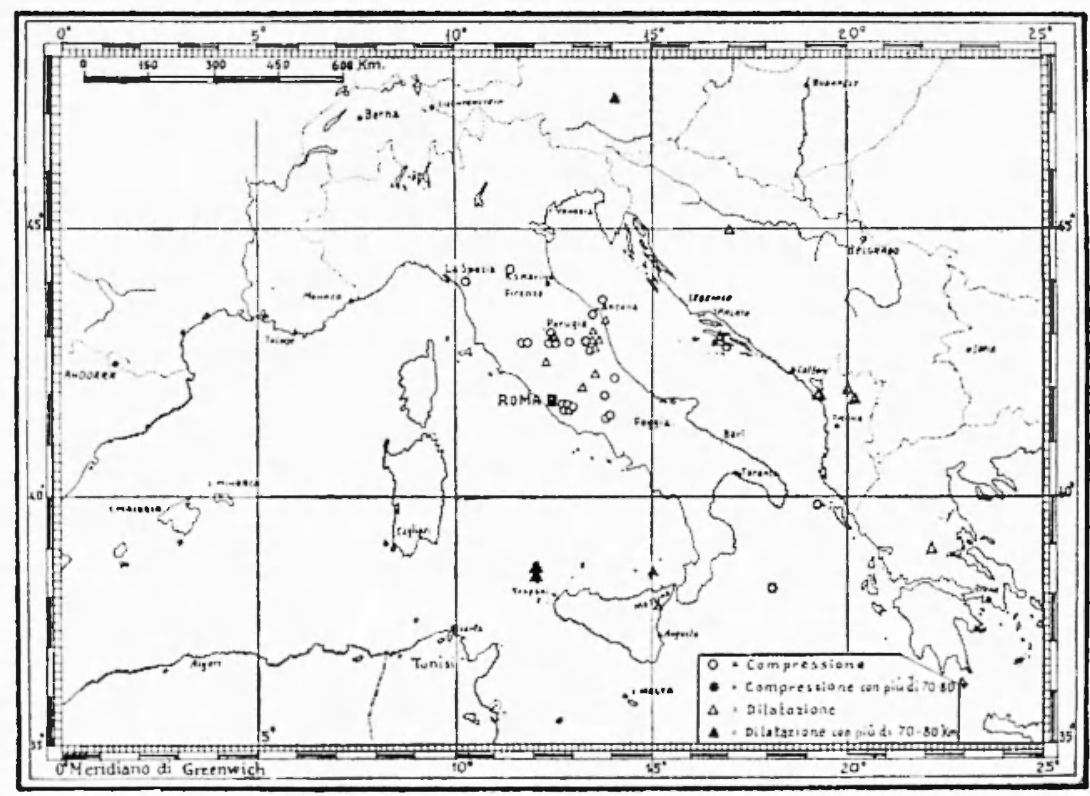

vial eccelto ma compressione in Damazia. Dallo Jonio, vicerersas arrivano compressioni.

Alemi dei terremoli del periodo in esame con epicentro in latlia, sono stati studiati in morlo aceuralo e per aleuni di essi si i anche poluta stabilire, con huona probahiliti, la natura della scossa all'ipocentro: cosi, per esempio, i lerremoti dell'll fehhraio 1939 Appennino losco-romarnolol ${ }^{+}{ }^{t}$, del 15 ottolie 1939 Garfagnanal ( $)$, del 19 giumo 1940 (Monte Amiala) ("i) furono dovuli a solle. vamento di strati, mentre il terremoto del 16 maro 1941 thasso Tirrenot $f^{i}$ e le scosse del Cansiglio dell'ottobre 1936 i? the nella tabella 11 non comprare perehé $\dot{e}$ relativo ad un periorlo diverso da quello da noi qui esaminatoi sono dovuti a frattura.

Roma _.. Istiluto Vazionale di Confisica - Giugno 1919. 


\section{RISSUNTO}

Viene fato uno studio statistico sulla natura dell'onda iniziale dei terremoti registrati a Roma nel periodo 10.33-19.13, con particolare riguardo all'lealia e alle zone immediatamenie vicine, fono ad una distanza di $1000 \mathrm{him}$ da Roma.

\section{BIBIIOCRAFI}

(1) O. Soxvunce, Sur la nuture de londe initiale des féléséismes enregistrés a Uccle de 1910 it 192\%. Tramux seientifiques, fase. 2, 1925, par. 65.

(a) E. Rotrí - E. Platiscrumtr, Nature des sécousses seismiques: compressions ef dilatations. 71 Congres des Societes Savantes. 1938, pag. 113-117.

(3) A. Lo Stmo, La registrazione e lo studio dei fenomeni sismici nell Istituto Nazionale di Geofisica del C.N.R. Rir. Scient., Amo XI, n. 10.

(' P. Citor, Il terrenoto dell Appennino tosco-romagnolo dell 71 febbraio 19.39. Ric. Scient. Anno X. n. 11.

15) E. Rosin, Il terremoto della Garfagnant del 15 oftobre 1939. Ric. Scient., Anno XI, n. $7-8$.

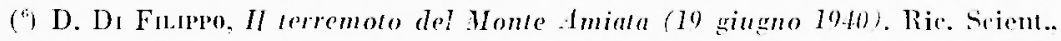
Anno XII, n. 7-8.9.

(๑) D. Dr Fiture. Studio microsismico del terremoto del basso Tirreno del 16 maro 19\%. Boll. Soc. Simol. Ital., vol. XXXIX, n. 3-t (1911).

(5) P. Cubor, Terremolo del Cansiglio dellothobre 1936. Ricerche su lerremoti al origine vicina. Rie. Scient., Inno IX, vol. II, n. i-ti. 
Tabela I

ISH MINORE - MIR EGEO

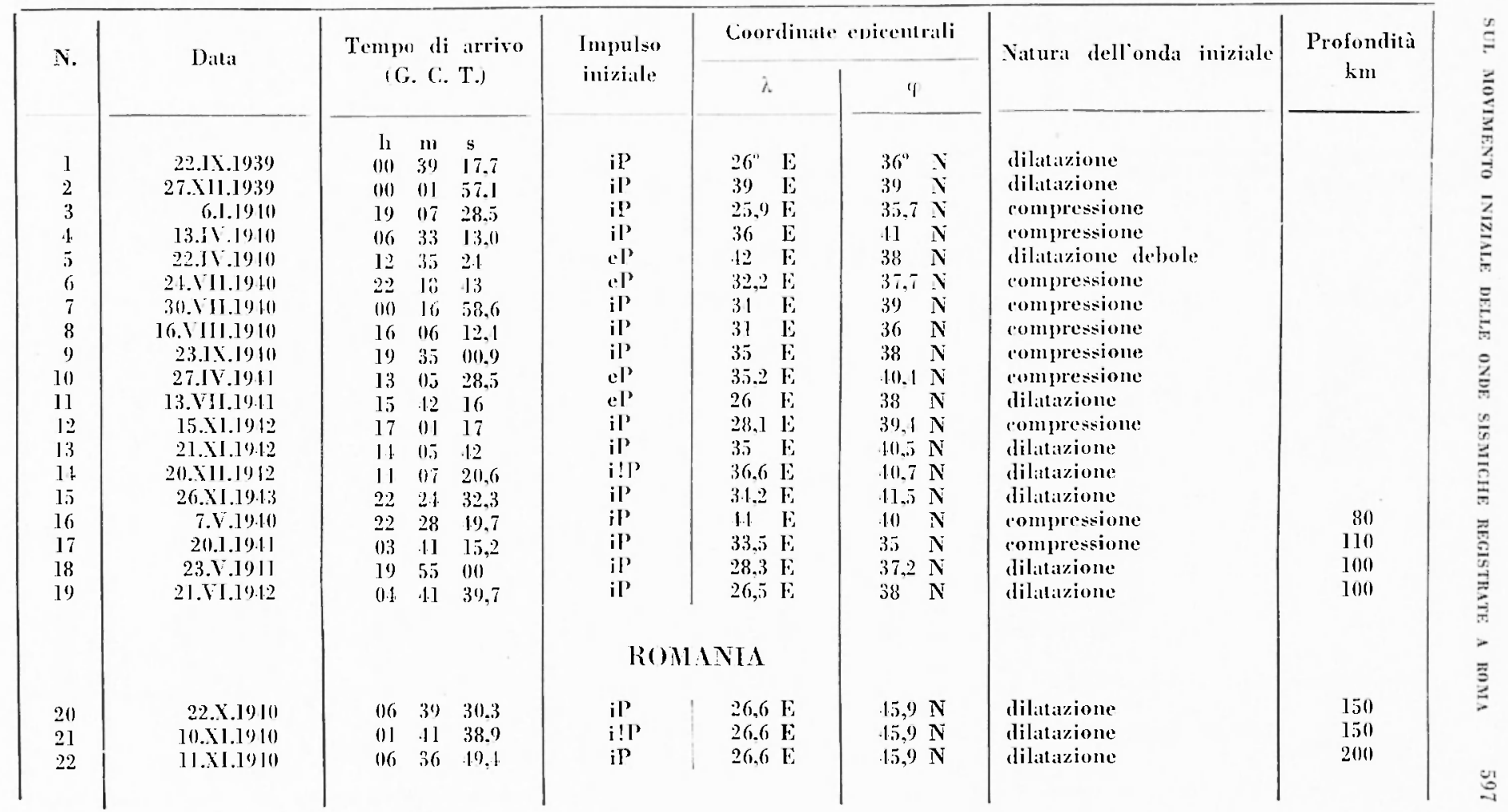




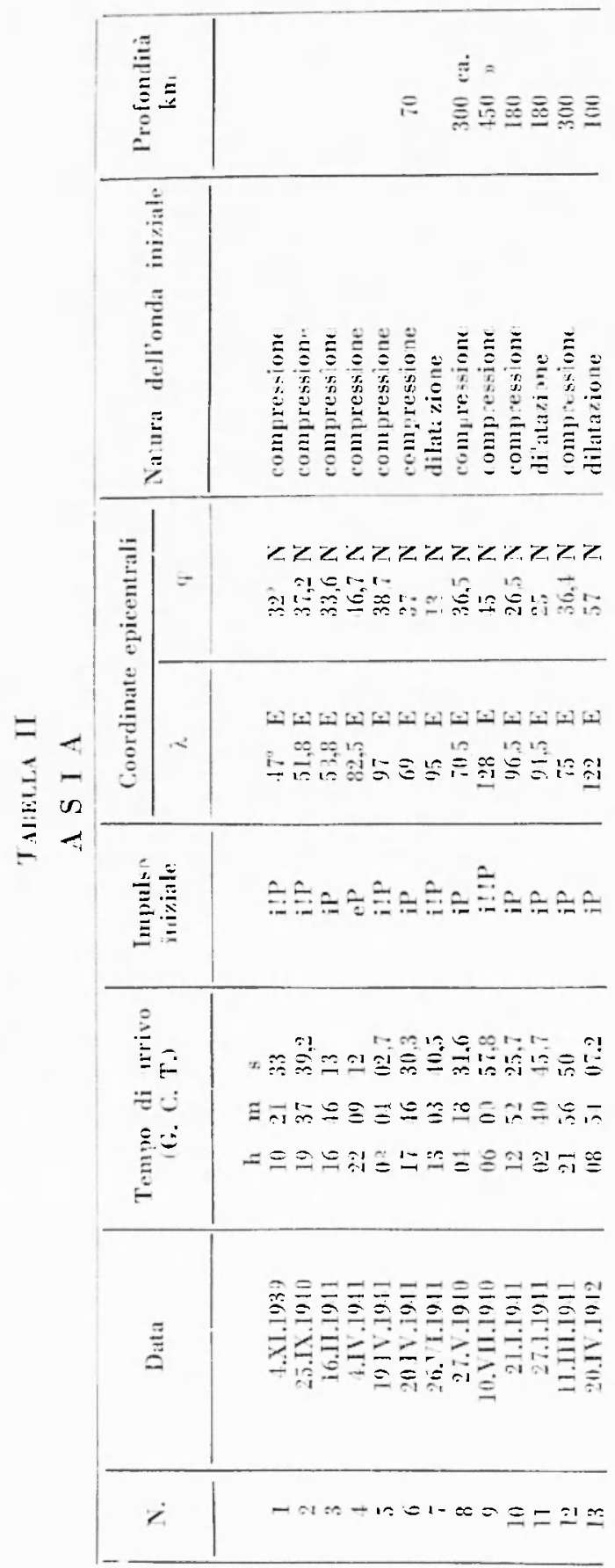


TaBella $1 \mathrm{~V}$

GIA P PONL

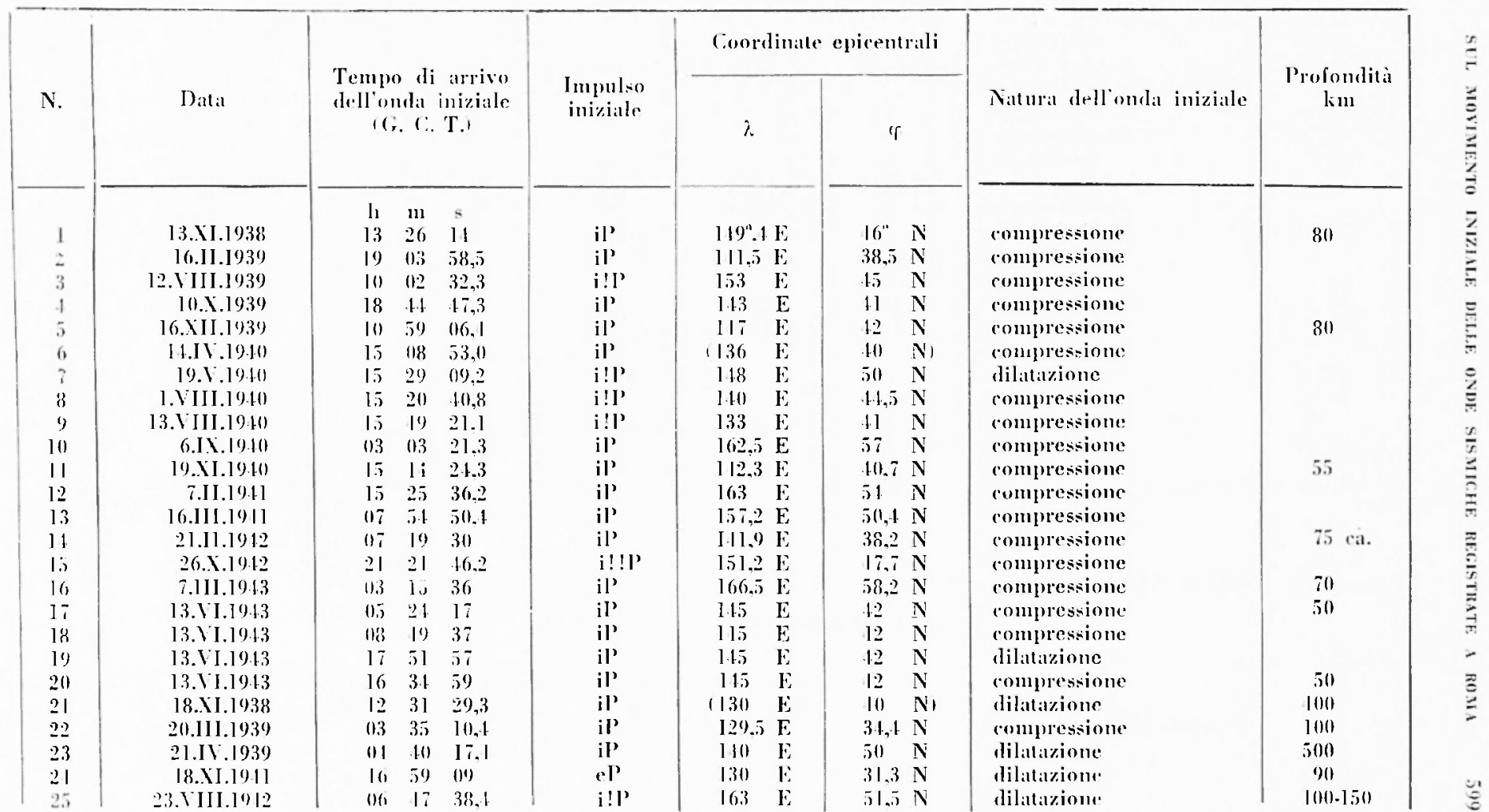




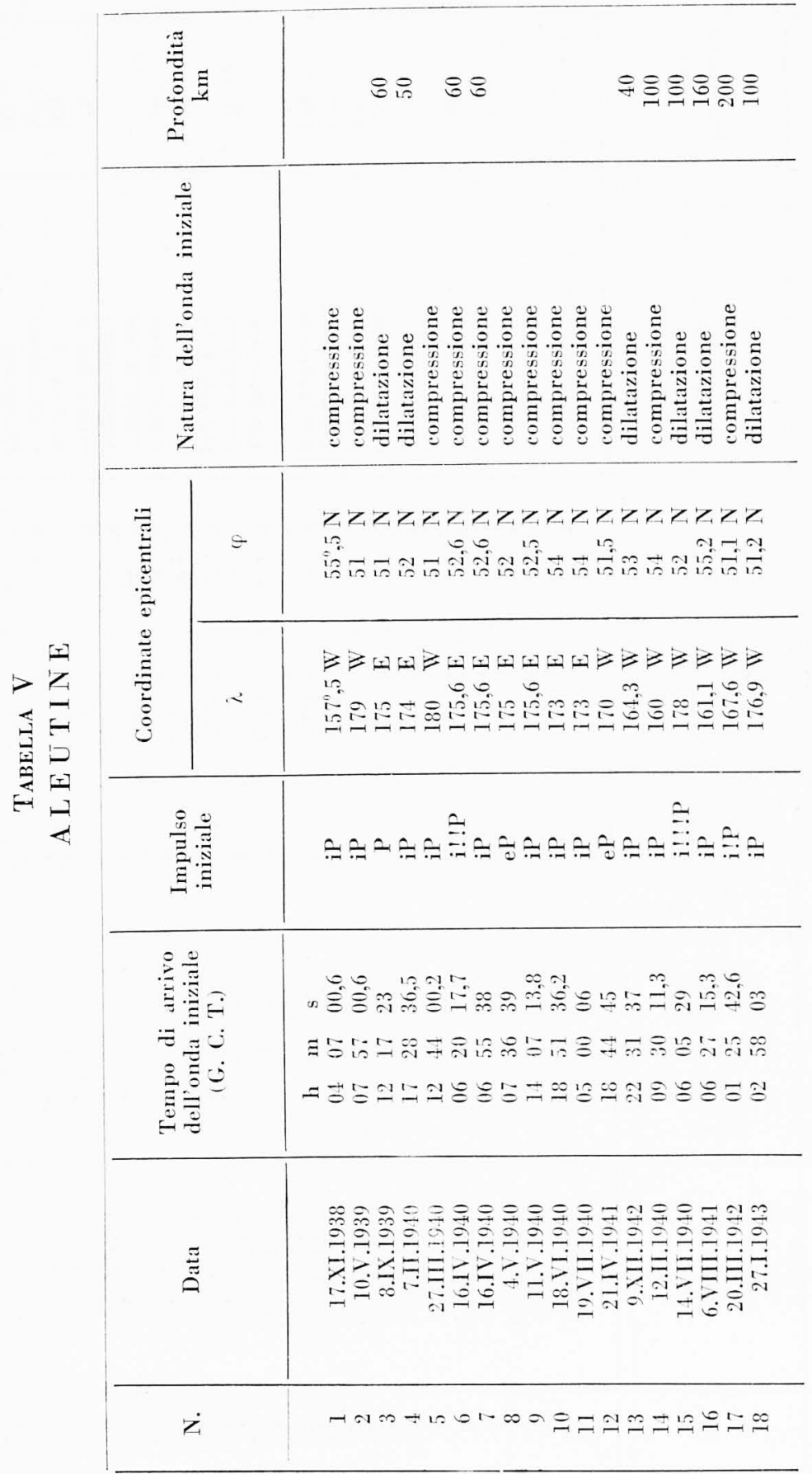


'TABRiLLA Vi

GRIPPO DELLE ISOLE

SOLONONE - NUONF EBRIDI - FIGII - TONGA - SAIOOA

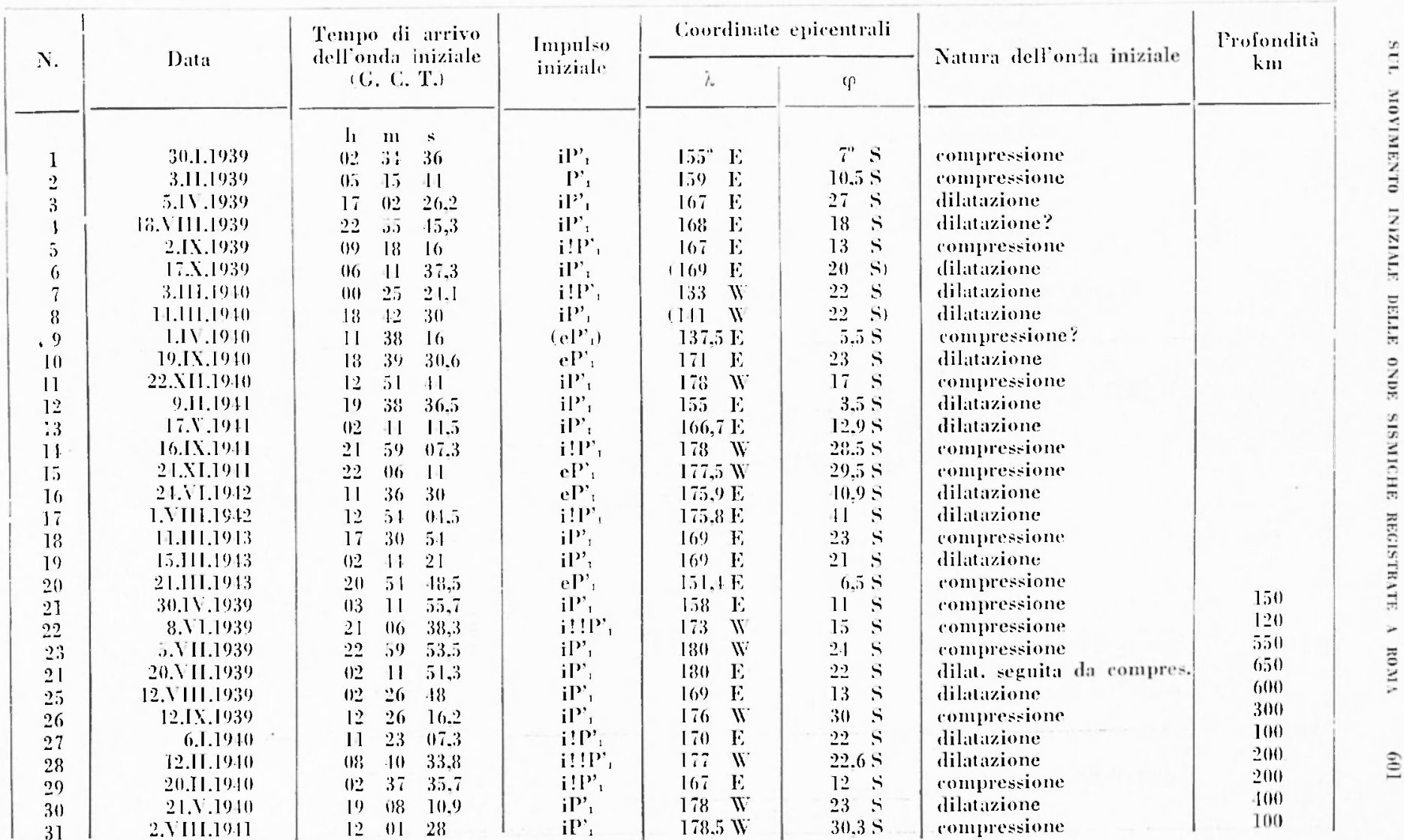


j'abElata Vll

AMERICA DEL NORD

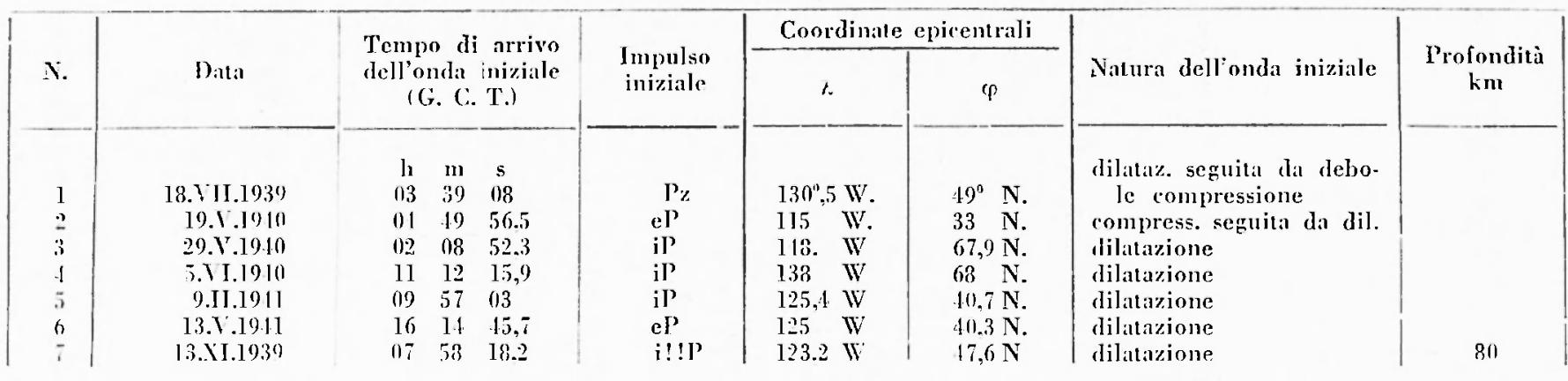

Tabella VIII

AMERICA CENTRALE - ANTILLE

\begin{tabular}{|c|c|c|c|c|c|c|c|c|c|}
\hline \multirow[b]{2}{*}{ N. } & \multirow[b]{2}{*}{ I)ital } & \multirow{2}{*}{\multicolumn{3}{|c|}{$\begin{array}{c}\text { Tempo di arrivo } \\
\text { dell'onda iniziale } \\
\text { (G. C. T.) }\end{array}$}} & \multirow{2}{*}{$\begin{array}{l}\text { Impulso } \\
\text { iniziale }\end{array}$} & \multicolumn{2}{|c|}{ Coordimate epicentrali } & \multirow[b]{2}{*}{ Natura dellonda iniziale } & \multirow{2}{*}{$\begin{array}{l}\text { Profondita } \\
\text { km }\end{array}$} \\
\hline & & & & & & $i$ & q & & \\
\hline 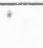 & & $l_{1}$ & nI & $\mathrm{s}$ & & & & & \\
\hline l & $28.1 \times .1939$ & 1.5 & 11 & 22 & el & $89 \mathrm{~W}$ & $14^{n}, 5 \mathrm{~N}$ & compressione debole & \\
\hline 2 & 21.111 .1030 & 21 & 07 & 17,1 & $\mathrm{i} ! ! \mathrm{P}$ & $85 \mathrm{~W}$ & $10 \mathrm{~N}$. & (ompressione & \\
\hline 3 & 20.111 .1939 & $0 !$ & 56 & 53,2 & $\mathrm{i} ! \mathrm{P}^{\mathrm{s}}$ & $85 \mathrm{~W}$ & $9.6 \mathrm{~N}$ & (compressione & \\
\hline .1 & 17.1 .1910 & 112 & 12 & 31 & $\mathrm{eP}$ & $32 \quad \mathrm{~W}$ & $7.9 \mathrm{~N}$ & compressione? & \\
\hline$\overline{5}$ & 13.151 .1910 & 17 & 00 & 20,1 & $\mathrm{i} ! \mathrm{P}^{3}$ & $63 \pi$ & $7.1 \mathrm{~N}$ & dilatazione & \\
\hline 0 & 5.x.1910 & $1 i$ & 1) & 10,6 & il & of W & $9 \mathrm{~N}$ & compressione & \\
\hline$i$ & T.JV.J111 & 23 & 11 & 26.1 & $\mathrm{eP}^{\mathrm{P}}$ & $70.3 \mathrm{~W}$ & $17.6 \mathrm{~N}$. & dilatarione & \\
\hline 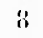 & 1.5 .11 .1911 & 19 & 23 & 17,7 & il' & $103 \mathrm{~W}$ & $18.8 \mathrm{~N}$. & compressione & \\
\hline 0 & 27.11 .1011 & 17 & 21 & 19.2 & il & $193 \mathrm{~W}$ & $16 N$ & ('ompressione & \\
\hline 10 & 6.1111 .1912 & 23 & 19 & 27 & il' & $90.9 \mathrm{WW}$ & $11.1 \mathrm{~N}$. & (ompressione & \\
\hline 11 & ล.1.J.794.3 & (b) & 11 & 18 & il' & $83 \mathrm{~W}$ & $5.5 \mathrm{~N}$ & dilatarione & \\
\hline $1: 2$ & I.YII.1"1:3 & 111 & (1) & 55 & $\mathrm{eP}^{3}$ & $31.5 \mathrm{~W}$ & $11.3 \times$ & compressione & \\
\hline 13 & $20.1 .1931)$ & $\therefore 0$ & 53 & 26.1 & iP & $91.5 \mathrm{~W}$ & $13 \mathrm{~N}$. & dilatazione & 450 \\
\hline 11 & 3.Xil.1939 & 08 & 13 & 11.1 & $\mathrm{i} ! \mathrm{P}$ & $92.5 \mathrm{~W}$ & $11.5 \mathrm{~N}$ & compressione & 75 \\
\hline 1.7 & 2.311 .1010 & i3 & $1 !$ & 311 & i! P & $91.3 \mathrm{~W}$ & $13.4 \mathrm{~N}$. & compressione & 100 \\
\hline 16 & $26 . X 11.1912$ & 12 & 11 & 11.7 & $P P$ & $\therefore \quad 11$ & $9.5 \mathrm{~N}$ & dilatazione & 190 \\
\hline 17 & 2.V.i9:3 & 17 & 29 & 57.6 & iP & $80.1 \mathrm{~W}$ & $7 \mathrm{~N}$. & dilatazione & 80 \\
\hline 18 & 15.VI.19.13 & 18 & 31 & 32 & il & $93,1 \mathrm{~W}$ & $13,7 \mathrm{~N}$. & compressione & 80 \\
\hline
\end{tabular}


Tabelat IX

AMERICA DEL SLD

\begin{tabular}{|c|c|c|c|c|c|c|c|c|c|}
\hline \multirow{2}{*}{ N. } & \multirow{2}{*}{ Data } & \multirow{2}{*}{\multicolumn{3}{|c|}{$\begin{array}{c}\text { Trumpo di arivo } \\
\text { dellonda iniziale } \\
\text { (C. C.. T.) }\end{array}$}} & \multirow{2}{*}{$\begin{array}{l}\text { Impulso } \\
\text { iniziale }\end{array}$} & \multicolumn{2}{|c|}{ Coordinate epicentrali } & \multirow{2}{*}{ Natura dell'onda iniziale } & \multirow{2}{*}{$\begin{array}{l}\text { Profonditi } \\
\text { knu }\end{array}$} \\
\hline & & & & & & $i$. & if & & \\
\hline 1 & $25 . I .1939$ & $\frac{11}{0.3}$ & $\begin{array}{l}m \\
16\end{array}$ & $\begin{array}{l}s \\
13.3\end{array}$ & $P$ & & $37^{n} S$ & conuressione & -30 \\
\hline 2 & 18.IV.1リ39 & 116 & 36 & 10,4 & $i P^{3}$ & $70 \mathrm{~W}$ & $27 \quad 5$ & compressione & 011 \\
\hline 3 & $4 . V I I .1939$ & 18 & 39 & 09,3 & eP & $67.3 \mathrm{~W}$ & $19,9 \mathrm{~S}$ & conturessione & \\
\hline 1 & 5.1 .1910 & 02 & 17 & 15,6 & $\mathrm{iP}^{3}$ & $8 \mathrm{I}, 1 \mathrm{~W}$ & $5.9 \mathrm{~S}$ & rompressione & 10 \\
\hline 5 & 2.1 .1 .1910 & 16 & 17 & 27,3 & $\mathrm{i} ! \mathrm{P}$ & $7 \pi .8 \mathrm{~W}$ & $10,8 \mathrm{~S}$. & comipressione & 511 \\
\hline 6 & 23.IX.19.10 & 117 & 27 & 10,9 & iP & $6.1 \mathrm{~W}$ & 215 & dilatizione & \\
\hline 7 & 1.X.1910 & 03 & 08 & 19 & $\mathrm{eP}$ & $67 \quad \mathrm{~W}$ & $19 \mathrm{~s}$ & dilatazione & \\
\hline 8 & 21.1111 .1912 & 23 & 01 & 03 & i] & $75,0 \mathrm{VI}$ & $11, \pi \mathrm{S}$ & compressione & \\
\hline ) & 6.711 .1910 & 113 & $\vdots 1$ & 12,9 & $\mathrm{i} ! ! \mathrm{P}$ & $61 \mathrm{~W}$ & $11 \mathrm{~N}$. & dilatazione & 175 \\
\hline 10 & 3.11 .1911 & 15 & 31 & 19.1 & $\mathrm{il}^{\mathrm{P}}$ & 69 & $25 \mathrm{~S}$ & complessione & $200 \mathrm{ca}$ \\
\hline 11 & 18.IX.1911 & 13 & 27 & 26.7 & $\mathrm{i} ! \mathrm{P}^{\mathrm{P}}$ & 73 & $13 \mathrm{~s}$. & dilatizione & 100 \\
\hline 12 & 11.1 .1912 & 02 & 26 & 30,7 & $\mathrm{iP}$ & $30 \quad \mathrm{~W}$ & $0.3 \mathrm{~S}$ & compressione & 500 \\
\hline 13 & 1.111 .1912 & 012 & 06 & 17 & $\mathrm{iP}$ & $80,2 \mathrm{~W}$ & $0.3 \mathrm{~N}$ & dilatizione & 500 \\
\hline 11 & 7.111 .1912 & 12 & 50 & .76 & $\mathrm{iP}^{3}$ & $(60,2 \mathrm{~W}$ & $0.3 \mathrm{~S}$ & dilatizione & $500 ?$ \\
\hline 15 & $8.1 \mathrm{IJ} .1912$ & 117 & 00 & 30 & $\mathrm{i} ! \mathrm{P}^{\mathrm{P}}$ & $69.5 \mathrm{~W}$ & $24.5 \mathrm{~s}$ & compressione & 175 \\
\hline 16 & $12.7 \mathrm{II} .1912$ & 15 & 18 & 29 & iP & $80,2 \mathrm{~W}$ & $0.3 \mathrm{~S}$ & complessione & $500 ?$ \\
\hline 18 & 12.XI.1912 & $1 . i$ & 39 & 36 & il' & $81.5 \mathrm{WV}$ & $0.0 \mathrm{~S}$ & compressione & 100 cal \\
\hline 17 & 30.1 .1913 & $0 . \overline{3}$ & 10 & 11 & iP & $80,2 \mathrm{~W}$ & 0,35 & dilatazione & 500 \\
\hline 19 & $6.1 \mathrm{~V} .19 .43$ & 16 & 21 & 27,4 & $\mathrm{iP}^{\mathrm{P}}$ & $71 \pi$ & $29.8 \mathrm{~S}$ & rompressione & 80 \\
\hline
\end{tabular}

Tabella $\mathrm{X}$

A F R I C A

\begin{tabular}{|c|c|c|c|c|c|c|c|}
\hline \multirow{2}{*}{ N. } & \multirow{2}{*}{ Data } & \multirow{2}{*}{$\begin{array}{c}\text { Tempo di arivo } \\
\text { dellonda iniziale } \\
\text { (G. (.. T.) }\end{array}$} & \multirow{2}{*}{$\begin{array}{l}\text { Intullso } \\
\text { inizials }\end{array}$} & \multicolumn{2}{|c|}{ Coordinate epicentrali } & \multirow{2}{*}{ Nallura dellonda inizinle } & \multirow{2}{*}{$\begin{array}{c}\text { Profomdità } \\
\text { km }\end{array}$} \\
\hline & & & & $\lambda$ & $\varphi$ & & \\
\hline $\begin{array}{l}1 \\
2 \\
3 \\
4\end{array}$ & $\begin{array}{r}20.1 .1939 \\
20 . \mathrm{I} .1939 \\
22 . \mathrm{VI} .1939 \\
19 . \mathrm{V} .191 .0\end{array}$ & $\begin{array}{ccc}11 & 111 & 5 \\
01 & 27 & 12.2 \\
11 & 25 & 11.6 \\
19 & 26 & 19.8 \\
18 & 27 & 25.6\end{array}$ & $\begin{array}{l}\mathrm{iP} \\
\mathrm{iP} \\
\mathrm{iP} \\
\mathrm{i} ! \mathrm{P}\end{array}$ & $\begin{aligned} & 1 15^{\prime \prime} \mathrm{E} \\
& 115 \mathrm{E} \\
& 1 \mathrm{~W} \\
& 35 \mathrm{E}\end{aligned}$ & 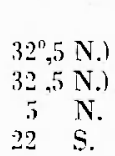 & $\begin{array}{l}\text { compressione } \\
\text { compressione } \\
\text { dilatazione } \\
\text { dilatazione }\end{array}$ & \\
\hline
\end{tabular}



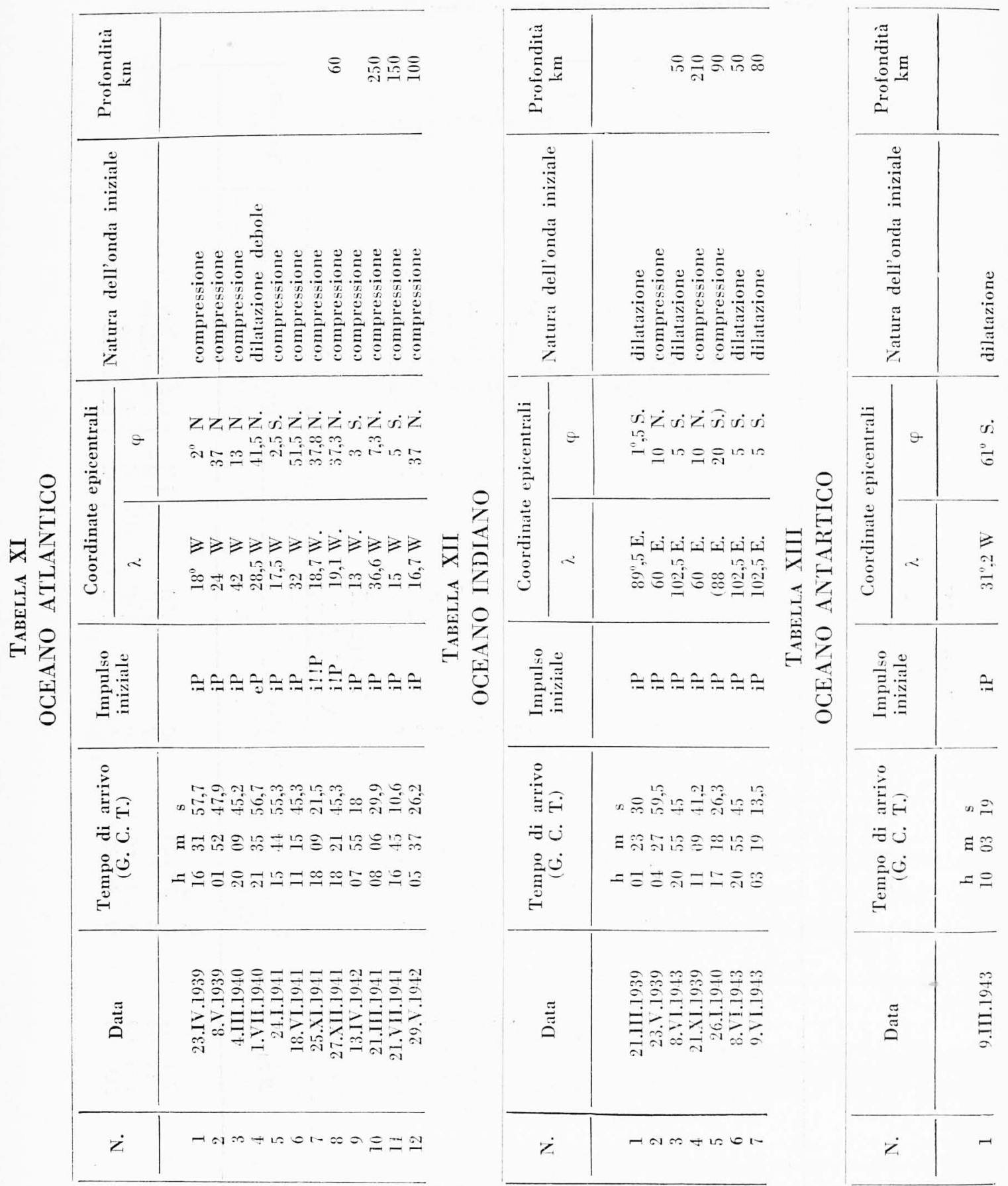
TABELL: XIY

ITALIA E DINTORN! ifmo a lo00 km da Roma!

\begin{tabular}{|c|c|c|c|c|c|c|c|c|c|c|c|c|c|c|c|}
\hline \multirow{2}{*}{ N. } & \multirow{2}{*}{ Data } & \multirow{2}{*}{\multicolumn{3}{|c|}{$\begin{array}{l}\text { Tempo } \\
\text { di arrivo } \\
\text { IC. C.. T.। }\end{array}$}} & \multirow{2}{*}{$\begin{array}{l}\text { Inpulso } \\
\text { iniziale }\end{array}$} & \multicolumn{5}{|c|}{ Coordinate seografiche } & & \multirow{2}{*}{ Zonil } & \multirow{2}{*}{$\begin{array}{l}\text { Distanzal } \\
\text { da Romil } \\
\text { km }\end{array}$} & \multirow{2}{*}{$\begin{array}{l}\text { Nalura della } \\
\text { onda iniziale }\end{array}$} & \multirow{2}{*}{$\begin{array}{l}E= \pm \\
\Xi=\end{array}$} \\
\hline & & & & & & & i. & & & If & & & & & \\
\hline 了 & $29 . \mathrm{XI} .1938$ & $\begin{array}{l}\text { l. } \\
22\end{array}$ & $\begin{array}{l}\text { In } \\
07\end{array}$ & 36,7 & il': & & -- & & & -- & & Colli Albani & 40 & wompressione & \\
\hline 2 & 1.XII.1938 & 22 & 52 & $2 \cdot 1,3$ & 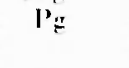 & $133^{\prime \prime}$ & 29 & $\mathrm{E}$ & $12 "$ & $8^{\prime}$ & $\mathbf{N}$ & $\begin{array}{l}\text { Sentilo a L'A. } \\
\text { quila }\end{array}$ & $9(0.95$ & dilatizione & \\
\hline 3 & $17 . \times 11.1938$ & 21 & 20 & $3.5,8$ & $P_{0}$ & & - & & & - & & Colli Albani & 28 & compressione & \\
\hline .1 & $26 . X I I .1938$ & (1) 1 & 22 & 15,6 & $\mathrm{l}_{\mathrm{n}}^{\mathrm{ir}}$ & & - & & & - & & Grotlaferrala & 30 & compressione & \\
\hline$\vec{\jmath}$ & 27.1 .1939 & 20 & Il & 16,9 & il' & $1.1 "$ & 53 & $\mathrm{E}$ & $38^{\prime \prime}$ & $28^{\prime}$ & $\mathrm{N}$ & Inole Lipari & 400 & dilalazione & \\
\hline 6 & 11.11 .1939 & 11 & 17 & 35 & $\mathbf{l}$ & $11 "$ & $39 '$ & $\mathrm{E}$ & 1.11 & 1 & $\mathbf{N}$ & $\begin{array}{l}\text { Marradi (Prov. } \\
\text { Bologna) }\end{array}$ & 250 & compressione & $5 \cdot 10$ \\
\hline 7 & 6.1 .1939 & 01 & 12 & 15,8 & $\mathrm{l}^{\prime}$ & $14^{\prime \prime}$ & & $\mathrm{E}$ & $17^{\prime \prime}: 2$ & & $\mathbf{N}$ & Passi Yauri & 600 & dilatarione & \\
\hline 8 & 20.1 .1939 & $(19)$ & 36 & 50,1 & $\Gamma^{\prime}$ & $19^{\prime \prime}, 2$ & & $\mathrm{E}$ & $11^{10} \div 9$ & & $\mathbf{N}$ & $\begin{array}{l}\text { Albania (pres. } \\
\text { so Sculari) }\end{array}$ & 550 & dilatasione & \\
\hline 9 & 11.VII.1939 & 03 & 17 & 53 & $P_{F}$ & $11 "$ & $1 \overrightarrow{5}$ & $\mathrm{E}$ & $42^{\circ}$ & $16^{\prime}$ & $N$ & Zonil C.bieti & 1.10 & compressione & \\
\hline 10 & 1.VIII.1939 & 13 & 15 & 22,1 & $P_{\underline{\underline{g}}}$ & $12^{11}$ & 33 & $\mathrm{~F}$ & $12^{11}$ & $32^{\prime}$ & $\mathbf{N}$ & Tona Narui & 70 & dilatizione & \\
\hline 31 & 9. VIII.1939 & $(1 ;)$ & 31 & 51,6 & I' & $(20)$ & 15, & $\mathrm{F}$ & $1.1^{\circ}$ & $50 \prime$ & N) & Albanial & 670 & dilislazione & \\
\hline 12 & A.IX.1939 & 07 & 13 & 53.9 & il'n & $13^{11}$ & 50 & $\mathrm{E}$ & $11^{\circ}$ & 26 & $\mathbf{N}$ & $\begin{array}{l}\text { Zonal di l'onle- } \\
\text { rorvo-Cassino }\end{array}$ & 130 & compressione & \\
\hline 13 & 9.IX.1939 & $0 \%$ & (i) & $1.0,6$ & il'm & $12^{\prime \prime}$ & $52 '$ & $\mathbf{E}$ & $1]^{\prime \prime}$ & 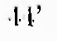 & $\mathbf{N}$ & Colli Nlbani & 3.5 ו & compressione & \\
\hline 14 & 20.IX.1939 & (1) & 21 & 29.1 & i!! In & $18 "$ & & E & $38^{\prime \prime}$ & & $\mathbf{N}$ & Miare Jomio & 760 & rmmpressione & \\
\hline $1 \overrightarrow{3}$ & $15 . \mathrm{X} .1939$ & 15 & 06 & 13.3 & $\mathrm{P}$ & $100^{\prime \prime}$ & $i=$ & $\mathbf{E}$ & 1.1. & 11 & $\mathrm{~N}$ & $\begin{array}{l}\text { Garfardiana } \\
\text { (Xlpi Ipuanies }\end{array}$ & 320 & compress. deh. & 26 \\
\hline 16 & $15 . X I I .1939$ & 00 & 57 & 13.8 & 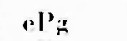 & $12 "$ & $301^{\prime}$ & $\mathbf{E}$ & $12^{\prime \prime}$ & 16 & $\mathbf{N}$ & Zonia Terni & 95 & rompressione & \\
\hline 17 & $15 . X I I .1939$ & 01 & 09 & 13.4 & $\mathrm{Cl}^{3} \mathrm{H}$ & $12^{\prime \prime}$ & $310^{\prime}$ & $\mathrm{F}$ & $12^{\prime \prime}$ & $16^{\circ}$ & $\mathrm{N}$ & Zonia Terni & 95 & rompressione & \\
\hline 18 & $15 . \times 11.1939$ & 01 & 29 & 35,1 & $\mathrm{il}^{\prime} \mathrm{g}$ & $12^{\prime \prime}$ & $300^{\prime}$ & $\mathbf{E}$ & $12^{\prime \prime}$ & $46^{\prime}$ & $\mathbf{N}$ & Zoma Terni & 95 & compressione & \\
\hline 19 & 23.11 .19 .10 & 00 & 41 & 19,8 & $\mathrm{il}^{5}$ & $19^{n}$ & $200^{\prime}$ & $\mathbf{E}$ & $39^{n}$ & $500^{\circ}$ & $\mathbf{N}$ & Cortin (Jonio) & 600 & romplessione & \\
\hline 20 & 19.1I.1910 & 11 & 10 & 33 & il's & $11^{\prime \prime}$ & 1.3 & $\mathbf{E}$ & $12^{n}$ & 5.3 & $\mathrm{~N}$ & Monle Amiala & 126 & rompressione & \\
\hline 21 & $27 . V I .1940$ & 09 & 27 & 32 & $\mathrm{eP}_{\mathrm{h}}$ & $112^{\prime \prime}$ & $15^{\prime}$ & $\mathbf{E}$ & $12^{\prime \prime}$ & 56 & $\mathbf{N})$ & 7oma Foligno & 110 & lompressione & \\
\hline 22 & $16 . X .1910$ & 13 & 17 & 57,6 & II!I'E & [1]" & $43^{\prime}$ & $\mathbf{E}$ & $1 \cdot 2^{\prime \prime}$ & $53^{\prime}$ & $\mathrm{N}$ & Nonte Amiata & 130 & rompressione & \\
\hline
\end{tabular}




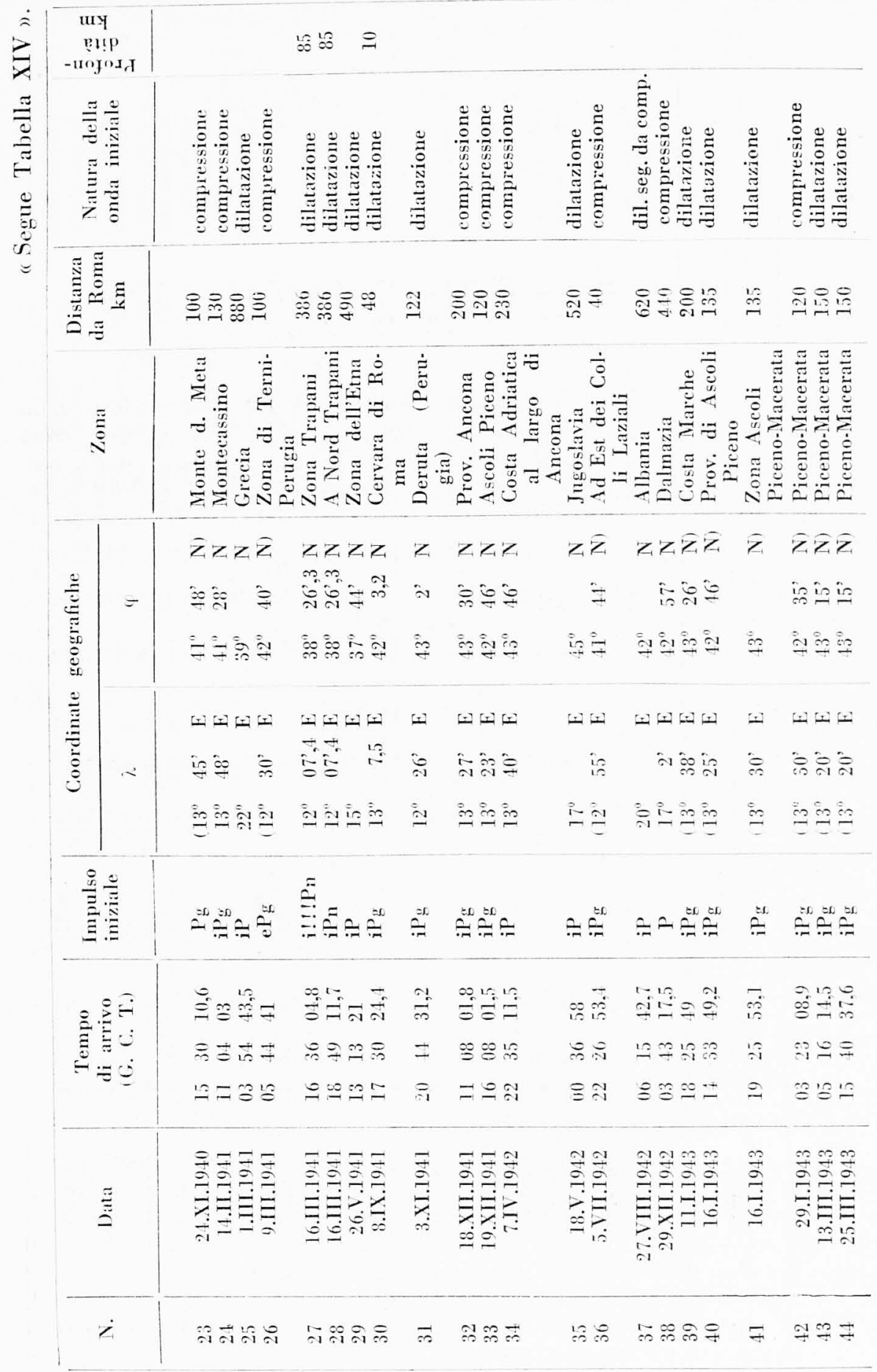

